May 2012

\title{
The Composite Higgs and Light Resonance Connection
}

\author{
Alex Pomarol ${ }^{a}$ and Francesco Riva ${ }^{b}$ \\ a Departament de Física, Universitat Autònoma de Barcelona, 08193 Bellaterra, Barcelona \\ b IFAE, Universitat Autònoma de Barcelona, 08193 Bellaterra, Barcelona
}

\begin{abstract}
Weinberg sum-rules have been used in the past to successfully predict the electromagnetic contribution to the charged-pion mass as a function of the meson masses. Following the same approach we calculate in the minimal composite Higgs model (MCHM) the Higgs mass as a function of the fermionic resonance masses. The simplicity of the method allows us to study several versions of the MCHM and show that a Higgs with a mass around $125 \mathrm{GeV}$ requires, quite generically, fermionic resonances below the TeV, and therefore accessible at the LHC. We also examine the couplings of the Higgs to the SM fermions and calculate their deviation from the SM value.
\end{abstract}




\section{Introduction}

LHC Higgs boson searches [1, 2] have cornered the Higgs into small mass windows. Combining these results with LEP [3], one can conclude, at 95\% CL, that the Higgs mass must lie in the narrow region $114.4 \mathrm{GeV}<m_{h}<127 \mathrm{GeV}$, or else it must be heavier than $\sim 600 \mathrm{GeV}$. More interestingly, the recently observed excess of events points towards a Higgs with $m_{h} \sim 125 \mathrm{GeV}$ and couplings (relatively) close to the SM values.

The existence of a light Higgs has important implications on models for the electroweak scale. The most interesting extensions of the SM in which a light Higgs is predicted are composite Higgs and supersymmetric models. In composite Higgs models the Higgs, similarly to pions in QCD, is light since it is a pseudo-Goldstone boson (PGB) arising from a new strong sector at TeV energies [4, 5]. Its mass is generated at the loop level through interactions with the SM states, which do not respect the (non-linearly realized) global symmetry which protects the Higgs mass. Since these loops are sensitive to the resonances of the strong sector, in these scenarios the Higgs mass is related to the mass of the heavy resonances. The exact relation is however difficult to calculate due to the intractable strong dynamics. One possibility to quantify this relation is to use AdS/CFT techniques [5, 6]. This has led to precise relations between the Higgs mass and the resonance masses, predicting that, in certain models, a light Higgs necessary requires fermionic resonances below the TeV [6]. Another possibility, recently explored, is to use deconstructed versions of the model, leading to similar conclusions [7, 8].

The purpose of this article is to analyze the relation between the Higgs mass and the fermionic resonance masses making use of the Weinberg sum-rules [9] which provide high-energy constraints on correlators (or spectral functions). In QCD, the Weinberg sum-rules, when combined with the assumption that only the lowest-mass resonances dominate the correlators, have led to important relations between physical quantities. One celebrated example is the electromagnetic contribution to the charged-pion mass [10], where a successful prediction was obtained, $m_{\pi^{+}}^{2}-m_{\pi^{0}}^{2} \simeq 3 \alpha m_{\rho}^{2} \log 2 /(2 \pi)$, relating the PGB mass to the resonance masses. Following a similar approach we will calculate the mass of a PGB Higgs. We will focus on the Minimal Composite Higgs model (MCHM) [5], generically defined as the model where the Higgs arises as a PGB from a strong sector whose global symmetry $\mathrm{SO}(5)$ is broken dynamically down to $\mathrm{SO}(4)$. Imposing the equivalent of the QCD Weinberg sum-rules in the large- $N_{c}$ limit, and assuming that the correlators are saturated by the lightest resonances, we will find simple expressions relating the Higgs mass to the fermionic resonance masses. The simplicity of the method will allow us to go beyond the minimal versions of the MCHM and study other scenarios. With the exception of very particular cases, we will show that a light Higgs necessarily implies fermionic resonances below the TeV, accessible to the LHC.

In section 2 we explain our procedure and show how to calculate the Higgs mass for the $\mathrm{MCHM}_{5}$ 
and $\mathrm{MCHM}_{10}$. In section 3 we extend this calculation to other MCHM and derive a generic lowerbound on the Higgs mass. In section 4 we summarize our results. In Appendix A we give the explicit relations between the top-quark form-factors and the correlators of the strong sector, while in Appendix B we give the effective lagrangian of the top in certain MCHM models of interest.

Note added: While this work was in preparation, Ref. [20] appeared, where the Weinberg sum-rules are also used to link the Higgs and fermion resonance masses and some of the formulas presented here are also derived.

\section{The Higgs mass in the MCHM}

In this section, we want to calculate the Higgs mass as a function of the resonance masses of the strong sector in different realizations of the MCHM. We will work in the unitary gauge where only the physical Higgs $h$ is kept and the SM Goldstones are gauged away. We start with the calculation of the gauge contribution to the Higgs potential, that follows closely the original calculation of the electromagnetic contribution to the charged-pion mass [10]. Then we compute the fermion contribution which, due to the large top-quark Yukawa coupling, is typically dominant.

\subsection{Gauge contributions to the Higgs potential}

Working in the limit $g^{\prime} \rightarrow 0$, the SM gauge contribution arising from loops of $\mathrm{SU}(2)_{L}$ gauge bosons is given by [5]

$$
V_{\text {gauge }}(h)=\frac{9}{2} \int \frac{d^{4} p}{(2 \pi)^{4}} \log \left(\Pi_{0}(p)+\frac{s_{h}^{2}}{4} \Pi_{1}(p)\right),
$$

where $s_{h} \equiv \sin h / f$, being $f$ the PGB decay-constant, and $p$ the Euclidian 4-momentum. We also have

$$
\Pi_{0}(p)=\frac{p^{2}}{g^{2}}+\Pi_{a}(p), \quad \Pi_{1}(p)=2\left[\Pi_{\hat{a}}(p)-\Pi_{a}(p)\right],
$$

where $g$ is the gauge coupling and $\Pi_{a}(p)$ is the two-point function of the $\mathrm{SO}(4)$ conserved current in momentum space, $\Pi_{a} \sim\left\langle J_{a} J_{a}\right\rangle$, and similarly $\Pi_{\hat{a}}$ for the current associated to the broken generators in $\mathrm{SO}(5) / \mathrm{SO}(4)$; for the precise definitions see Ref. [5]. In a large- $N$ expansion, that we will assume here, these form factors can be written as an infinite sum over narrow resonances:

$$
\Pi_{a}(p)=p^{2} \sum_{n} \frac{F_{\rho_{n}}^{2}}{p^{2}+m_{\rho_{n}}^{2}}, \quad \Pi_{\hat{a}}(p)=p^{2} \sum_{n} \frac{F_{a_{n}}^{2}}{p^{2}+m_{a_{n}}^{2}}+\frac{1}{2} f^{2},
$$

where $\rho_{n}$ and $a_{n}$ are vector resonances coming respectively in 6-plets and 4-plets of SO(4), and $F_{\rho_{n}, a_{n}}$ are referred to as the decay-constants of these resonances. 
The Higgs-dependent part of the potential Eq. (1) is expected to be finite. Indeed, according to the operator product expansion, the form factor $\Pi_{1}(p)$ must drop at large $p$ as $\sim\langle\mathcal{O}\rangle / p^{d-2}$, where $\mathcal{O}$ is the lowest dimension $d$ operator of the strong sector responsible for the $\mathrm{SO}(5) \rightarrow \mathrm{SO}(4)$ breaking. In large- $N_{c} \mathrm{QCD}$, in the limit of massless quarks, we have $\langle\mathcal{O}\rangle \sim\langle q \bar{q}\rangle^{2}$ and then $d=6$, with the left-right correlator $\Pi_{L R}(p)=\Pi_{V}-\Pi_{A} \rightarrow\langle q \bar{q}\rangle^{2} / p^{4}$ being the equivalent of our $\Pi_{1}(p)$. We assume that in the TeV strong sector $d>4$, meaning that the integral $\int d^{4} p \Pi_{1}(p) / \Pi_{0}(p)$ is convergent for $\Pi_{0} \sim p^{2}$, assuring the finiteness of the Higgs-dependent part of the potential Eq. (1). This convergence is equivalent to imposing a set of requirements on $\Pi_{1}(p)$, usually known as the Weinberg sum-rules [9]. These are

$$
\lim _{p^{2} \rightarrow \infty} \Pi_{1}(p)=0, \quad \quad \lim _{p^{2} \rightarrow \infty} p^{2} \Pi_{1}(p)=0,
$$

that give two constraints to be fulfilled by the decay constants and masses in Eq. (3). Following Ref. [10], we can now make the extra assumption of truncating the infinite sum in Eq. (3) to include only the minimal number of resonances needed to satisfy the sum-rules Eq. (4). One can easily realize that only two are needed, $\rho_{1} \equiv \rho$ and $a_{1}$. Using the two constraints Eq. (4) we can determine $F_{\rho}$ and $F_{a_{1}}$, and then calculate $\Pi_{1}$ as a function of the two resonance masses ${ }^{1}$.

$$
\Pi_{1}(p)=\frac{f^{2} m_{\rho}^{2} m_{a_{1}}^{2}}{\left(p^{2}+m_{\rho}^{2}\right)\left(p^{2}+m_{a_{1}}^{2}\right)} .
$$

Eq. (5) can now be used to obtain the gauge contribution to the Higgs potential Eq. (1). In an expansion $g^{2} \ll 1$, we have

$$
V(h)=\alpha s_{h}^{2}+\beta s_{h}^{4}+\cdots,
$$

where

$$
\begin{aligned}
\alpha= & \frac{9 g^{2} f^{2} m_{\rho}^{2} m_{a_{1}}^{2}}{128 \pi^{2}\left(m_{a_{1}}^{2}-m_{\rho}^{2}\right)} \log \left(\frac{m_{a_{1}}^{2}}{m_{\rho}^{2}}\right) \\
\beta=-\frac{9 g^{4} f^{4}}{1024 \pi^{2}}[ & \log \left(\frac{m_{a_{1}} m_{\rho}}{m_{W}^{2}}\right)-\frac{\left(m_{a_{1}}^{4}+m_{\rho}^{4}\right)}{\left(m_{a_{1}}^{2}-m_{\rho}^{2}\right)^{2}} \\
& \left.\quad-\frac{\left(m_{a_{1}}^{2}+m_{\rho}^{2}\right)\left(m_{a_{1}}^{4}-4 m_{a_{1}}^{2} m_{\rho}^{2}+m_{\rho}^{4}\right)}{2\left(m_{a_{1}}^{2}-m_{\rho}^{2}\right)^{3}} \log \left(\frac{m_{a_{1}}^{2}}{m_{\rho}^{2}}\right)\right]
\end{aligned}
$$

and in the calculation of $\beta$ the infrared divergence has been regularized with the $W$ mass. Notice that, being $\alpha$ positive, the gauge contribution alone cannot induce electroweak symmetry breaking (EWSB).

\footnotetext{
${ }^{1}$ This result is straightforward to obtain in the following alternative way. Requiring that $\Pi_{1}$ has two poles corresponding to the two massive resonances implies that the denominator of $\Pi_{1}$ must be $\left(p^{2}+m_{\rho}^{2}\right)\left(p^{2}+m_{a_{1}}^{2}\right)$; the numerator can easily be obtained by requiring $\Pi_{1}(0)=f^{2}$.
} 


\subsection{Top contributions in the $\mathrm{MCHM}_{5}$}

We can now repeat the same procedure for the fermionic contributions to the Higgs potential, concentrating on the one from the top quark, which is usually the most important one and generates a Higgs potential with an EWSB minimum.

As in Ref. [5], we will consider models in which the SM fermions couple to the strong sector by mixing with fermionic operators. These mixings are defined by the embedding of the SM fermions into $\mathrm{SO}(5)$ spurion fields (see Appendix A). In this section we will work in the $\mathrm{MCHM}_{5}$ [6] where the left-handed and right-handed top, $t_{L}$ and $t_{R}$, are respectively embedded in two spurions in the $\mathbf{r}_{\mathbf{L}}=\mathbf{5}$ and $\mathbf{r}_{\mathbf{R}}=\mathbf{5}$ representation of $\mathrm{SO}(5)$. The (non-local) effective theory for the top quark, at the quadratic order, can be written in momentum space as

$$
\mathcal{L}_{\mathrm{eff}}=\bar{t}_{L} \not p\left(\Pi_{0}^{t_{L}}(p)+\frac{s_{h}^{2}}{2} \Pi_{1}^{t_{L}}(p)\right) t_{L}+\bar{t}_{R} \not p\left(\Pi_{0}^{t_{R}}(p)+c_{h}^{2} \Pi_{1}^{t_{R}}(p)\right) t_{R}+\left(\frac{s_{h} c_{h}}{\sqrt{2}} \bar{t}_{L} M_{1}^{t}(p) t_{R}+\text { h.c. }\right),
$$

where the form factors $\Pi_{0,1}^{t_{L, R}}(p)$ and $M_{1}^{t}(p)$ encode the strong sector dynamics. The top contribution to the Higgs potential is then [6] $]^{2}$

$$
V_{\text {top }}(h)=-2 N_{c} \int \frac{d^{4} p}{(2 \pi)^{4}} \log \left[-p^{2}\left(\Pi_{0}^{t_{L}}+\frac{s_{h}^{2}}{2} \Pi_{1}^{t_{L}}\right)\left(\Pi_{0}^{t_{R}}+c_{h}^{2} \Pi_{1}^{t_{R}}\right)-\frac{s_{h}^{2} c_{h}^{2}}{2}\left|M_{1}^{t}\right|^{2}\right],
$$

where $N_{c}=3$ and, as shown in Appendix A, the top-quark form factors can be written as a function of the correlators of the fermionic operators decomposed in $\mathrm{SO}(4)$-representations:

$$
\begin{array}{lll}
\Pi_{0}^{t_{L}}(p)=1+\Pi_{Q_{4}}^{L}(p), & \Pi_{1}^{t_{L}}(p)=\Pi_{Q_{1}}^{L}(p)-\Pi_{Q_{4}}^{L}(p), \\
\Pi_{0}^{t_{R}}(p)=1+\Pi_{Q_{4}}^{R}(p), & \Pi_{1}^{t_{R}}(p)=\Pi_{Q_{1}}^{R}(p)-\Pi_{Q_{4}}^{R}(p), \\
M_{1}^{t}(p)=M_{Q_{1}}(p)-M_{Q_{4}}(p) . &
\end{array}
$$

Notice that we have canonically normalized the kinetic term of the top in the limit in which the top decouples from the strong sector. As in the case of the gauge correlators, $\Pi_{Q_{4,1}}^{L, R}$ and $M_{Q_{4,1}}$ can be written in a large- $N$ expansion as a sum over infinite resonances. We have

$$
\Pi_{Q_{4}}^{L}(p)=\sum_{n} \frac{\left|F_{Q_{4}^{(n)}}^{L}\right|^{2}}{p^{2}+m_{Q_{4}^{(n)}}^{2}}, \quad \Pi_{Q_{1}}^{L}(p)=\sum_{n} \frac{\left|F_{Q_{1}^{(n)}}^{L}\right|^{2}}{p^{2}+m_{Q_{1}^{(n)}}^{2}},
$$

and similarly for $\Pi_{Q_{4,1}}^{R}$ with the replacement $L \rightarrow R$, while

$$
M_{Q_{4}}(p)=\sum_{n} \frac{F_{Q_{4}^{(n)}}^{L} F_{Q_{4}^{(n)}}^{R *} m_{Q_{4}^{(n)}}}{p^{2}+m_{Q_{4}^{(n)}}^{2}}, \quad M_{Q_{1}}(p)=\sum_{n} \frac{F_{Q_{1}^{(n)}}^{L} F_{Q_{1}^{(n)}}^{R *} m_{Q_{1}^{(n)}}}{p^{2}+m_{Q_{1}^{(n)}}^{2}} .
$$

\footnotetext{
${ }^{2}$ We are working in a large- $N$ expansion and neglect contributions coming from form factors involving four or more top-quarks.
} 
We denote with $Q_{4}^{(n)}$ and $Q_{1}^{(n)}$ the (color-triplet) vector-like fermonic resonances with the $\operatorname{SO}(4)$ quantum numbers of $\mathbf{4}$ and $\mathbf{1}$ respectively. The dimensionful parameters $F_{Q_{i}^{(n)}}^{L, R}$ are the equivalent of the decay constants of spin-1 resonances but here for fermions: they correspond to mixing massterms between the top quark and the fermionic resonances. For convenience we define the mixing parameter

$$
F_{Q_{i}^{(n)}}^{L, R} / m_{Q_{i}^{(n)}} \equiv \epsilon
$$

As with the gauge contribution, we want to calculate the correlators of Eq. (11) considering only the minimal number of resonances necessary for the convergence of the Higgs potential Eq. 10. To obtain a finite result we need that at large momentum the form factors $\Pi_{1}^{t_{L, R}}$ fall off at least as $\Pi_{1}^{t_{L, R}} \rightarrow 1 / p^{6}$. This is equivalent to imposing three pairs of Weinberg sum-rules, $\lim _{p \rightarrow \infty} p^{n} \Pi_{1}^{t_{L, R}}(p)=0(n=0,2,4)$ that can be fulfilled with at least three vector-like resonances. Our main interest, however, is in the calculation of the Higgs mass which, as we will now show, requires less resonances to be finite.

Let us calculate the Higgs mass in the approximation of small Higgs vacuum expectation value $\left\langle s_{h}\right\rangle \ll 1$. The potential Eq. (10) can be expanded as

$$
V(h)=\alpha s_{h}^{2}-\beta s_{h}^{2} c_{h}^{2}+\cdots,
$$

where $\alpha=O\left(\epsilon^{2}\right)$ and $\beta=O\left(\epsilon^{4}\right)$. Notice that at least two terms must be included in the expansion in order to have realistic EWSB. Indeed, for $\alpha<\beta$ and $\beta \geqslant 0$ we have that the electroweak symmetry is broken:

$$
\left\langle s_{h}\right\rangle \equiv \frac{v}{f} \equiv \sqrt{\xi} \simeq \sqrt{\frac{\beta-\alpha}{2 \beta}}
$$

and the Higgs mass is given by

$$
m_{h}^{2} \simeq \frac{8 \beta}{f^{2}}\left\langle s_{h}^{2} c_{h}^{2}\right\rangle
$$

with

$$
\beta=N_{c} \int \frac{d^{4} p}{(2 \pi)^{4}}\left[\frac{\left|M_{1}^{t}\right|^{2}}{p^{2} \Pi_{0}^{t_{L}} \tilde{\Pi}_{0}^{t_{R}}}+\left(\frac{\Pi_{1}^{t_{L}}}{2 \Pi_{0}^{t_{L}}}\right)^{2}+\left(\frac{\Pi_{1}^{t_{R}}}{\tilde{\Pi}_{0}^{t_{R}}}\right)^{2}\right]
$$

where $\tilde{\Pi}_{0}^{t_{R}} \equiv \Pi_{0}^{t_{R}}+\Pi_{1}^{t_{R}}$. If instead of an expansion in $\left\langle s_{h}^{2}\right\rangle$, we had performed an expansion in $\epsilon^{2}$, we would still have obtained Eqs. 16 - 18 , but with $\Pi_{0}^{t_{L}}=\tilde{\Pi}_{0}^{t_{R}} \simeq 1$. Let us from now on work in this limit, $\epsilon^{2} \ll 1$, corresponding to small mixing between the top and the resonances; we will see later that this is a good approximation for most of our calculations. From Eq. (18) we can easily derive a lower-bound on the Higgs mass as a function of the lightest resonance mass. This is based on the fact that the three terms in Eq. (18) are positive, meaning that we can bound the Higgs mass using only the first one:

$$
m_{h}^{2} \geq \frac{2 N_{c}}{\pi^{2}} \frac{m_{t}^{2}}{f^{2}} \int_{0}^{\infty} d p p\left|\frac{M_{1}^{t}(p)}{M_{1}^{t}(0)}\right|^{2}
$$


where we have used the fact that the physical top mass is given by

$$
m_{t}=\frac{\left|M_{1}^{t}(0)\right|}{\sqrt{2 \Pi_{0}^{t_{L}}(0) \tilde{\Pi}_{0}^{t_{R}}(0)}}\left\langle s_{h} c_{h}\right\rangle .
$$

The convergence of Eq. (19) requires the Weinberg sum-rule $\lim _{p \rightarrow \infty} M_{1}^{t}(p)=0$. This can be achieved with just one resonance,

$$
\left|\frac{M_{1}^{t}(p)}{M_{1}^{t}(0)}\right|=\frac{m_{Q}^{2}}{p^{2}+m_{Q}^{2}},
$$

where $Q$ represents here the lightest resonance, that can either be a 4 or a $\mathbf{1}$ of $\mathrm{SO}(4)$, since this procedure does not depend on its quantum numbers. We then have

$$
m_{h}^{2} \geq \frac{N_{c}}{\pi^{2}} \frac{m_{t}^{2}}{f^{2}} m_{Q}^{2}
$$

that provides an upper bound for the resonance mass:

$$
m_{Q} \lesssim 700 \mathrm{GeV}\left(\frac{m_{h}}{125 \mathrm{GeV}}\right)\left(\frac{160 \mathrm{GeV}}{m_{t}}\right)\left(\frac{f}{500 \mathrm{GeV}}\right) .
$$

To obtain a convergent result for the Higgs mass from the full top-quark contribution of Eq. (18), we must impose the two pairs of Weinberg sum-rules, $\lim _{p \rightarrow \infty} p^{n} \Pi_{1}^{t_{L, R}}(p)=0(n=0,2)$, that require at least two resonances, $Q_{1}^{(1)} \equiv Q_{1}$ and $Q_{1}^{(4)} \equiv Q_{4}$. We obtain

$$
\begin{aligned}
\Pi_{1}^{t_{L, R}} & =\left|F_{Q_{4}}^{L, R}\right|^{2} \frac{\left(m_{Q_{4}}^{2}-m_{Q_{1}}^{2}\right)}{\left(p^{2}+m_{Q_{4}}^{2}\right)\left(p^{2}+m_{Q_{1}}^{2}\right)}, \\
M_{1}^{t}(p) & =\left|F_{Q_{4}}^{L} F_{Q_{4}}^{R *}\right| \frac{m_{Q_{4}} m_{Q_{1}}\left(m_{Q_{4}}-m_{Q_{1}} e^{i \theta}\right)}{\left(p^{2}+m_{Q_{4}}^{2}\right)\left(p^{2}+m_{Q_{1}}^{2}\right)}\left(1+\frac{p^{2}}{m_{Q_{4}} m_{Q_{1}}} \frac{m_{Q_{1}}-m_{Q_{4}} e^{i \theta}}{m_{Q_{4}}-m_{Q_{1}} e^{i \theta}}\right),
\end{aligned}
$$

where we have defined $F_{Q_{4}}^{L} F_{Q_{4}}^{R *}=e^{i \theta}\left|F_{Q_{4}}^{L} F_{Q_{4}}^{R *}\right|$ and set by a field redefinition $F_{Q_{1}}^{L} F_{Q_{1}}^{R}$ to be real. Eq. (24) together with Eq. (20) gives ${ }^{3}$

$$
m_{h}^{2} \simeq \frac{N_{c}}{\pi^{2}}\left[\frac{m_{t}^{2}}{f^{2}} \frac{m_{Q_{4}}^{2} m_{Q_{1}}^{2}}{m_{Q_{1}}^{2}-m_{Q_{4}}^{2}} \log \left(\frac{m_{Q_{1}}^{2}}{m_{Q_{4}}^{2}}\right)+\frac{\left(\Delta F^{2}\right)^{2}}{4 f^{2}}\left\langle s_{h}^{2} c_{h}^{2}\right\rangle\left(\frac{1}{2} \frac{m_{Q_{4}}^{2}+m_{Q_{1}}^{2}}{m_{Q_{1}}^{2}-m_{Q_{4}}^{2}} \log \left(\frac{m_{Q_{1}}^{2}}{m_{Q_{4}}^{2}}\right)-1\right)\right]
$$

where $\Delta F^{2}=\left|F_{Q_{4}}^{L}\right|^{2}-2\left|F_{Q_{4}}^{R}\right|^{2}$. It is easy to see that the second term in Eq. (25) is always positive and that the first term minimizes for $m_{Q_{4}} \rightarrow m_{Q_{1}}$ where the Higgs mass saturates the lower-bound Eq. (22). It is also important to notice that, considering only the top contributions to the Higgs potential, one obtains that $\alpha$ in Eq. (15) is proportional to $\Delta F^{2}$, meaning that the condition $\alpha<\beta$ requires small values for $\Delta F^{2}$. In this limit, the Higgs mass comes entirely from the first term of Eq. (25). In Figure 1 we show the value of the two lightest resonance masses for a Higgs mass

\footnotetext{
${ }^{3} \mathrm{~A}$ similar expression has also been obtained in the context of deconstructed MCHM [7].
} 


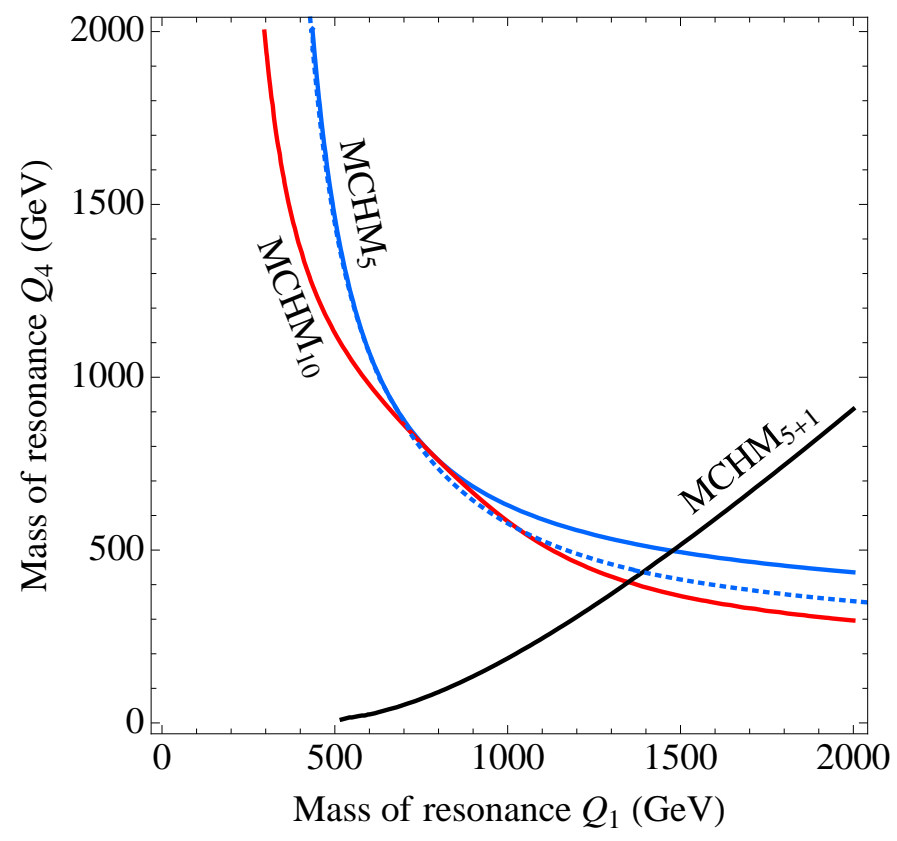

Figure 1: Masses of the two lightest fermion resonances for $m_{h}=125 \mathrm{GeV}$ (taking $\xi=0.2$ and $m_{t}=160 \mathrm{GeV}$ (the running top mass at $\sim \mathrm{TeV})$ ). In blue we plot the $\mathrm{MCHM}_{5}$ result; the solid line corresponds to Eq. (25) calculated in the approximation $\epsilon^{2} \ll 1$, while the dashed line is the exact result (always $\Delta F^{2}=0$ ). In solid red we plot the result for the $M_{C H} M_{10}\left(\epsilon^{2} \ll 1\right.$ and $\left.\Delta F^{2}=0\right)$ with $Q_{1} \rightarrow Q_{6}$. The black solid line is for $\mathbf{r}_{\mathbf{L}}=\mathbf{5}$ and $\mathbf{r}_{\mathbf{R}}=\mathbf{1}$ (denoted $\left.M C H M_{5+1}\right)$, fixing for illustration $F_{Q_{1}}^{L}=\sqrt{2} \tilde{F}_{Q_{1}}^{R}$.

$m_{h}=125 \mathrm{GeV}$. One can see that there is always a light state with a mass roughly between $500 \mathrm{GeV}$ and $700 \mathrm{GeV}$. Now, since light resonances imply large values of $\epsilon$ (see Eq. (14)), one could worry about the validity of our approximation $\epsilon^{2} \ll 1$. In Figure 1 we show with a dashed blue line the result obtained without taking the small $\epsilon^{2}$ limit. As can be appreciated, the differences are small and the approximation $\epsilon^{2} \ll 1$ always gives a more conservative upper-bound on the resonance masses. We note however that in the exact result the masses of the lightest fermionic resonances differ from $m_{Q_{1,4}}$ due to the sizable mixings with $t_{L, R}$. Therefore not only a light Higgs implies light fermionic resonances, but also a sizable degree of compositeness of the top.

A very similar model to the $\mathrm{MCHM}_{5}$ is the $\mathrm{MCHM}_{10}[6$, in which the left-handed and righthanded top quarks are embedded into spurions in the $\mathbf{1 0}$ representation of $\mathrm{SO}(5)$. In this model Eqs. (15)-17) also hold, and $\beta$ is given by 4

$$
\beta=N_{c} \int \frac{d^{4} p}{(2 \pi)^{4}}\left[\frac{\left|M_{1}^{t}\right|^{2}}{8 p^{2} \Pi_{0}^{t_{L}} \Pi_{0}^{t_{R}}}+\left(\frac{3 \Pi_{1}^{t_{L}}}{4 \Pi_{0}^{t_{L}}}\right)^{2}+\left(\frac{\Pi_{1}^{t_{R}}}{4 \Pi_{0}^{t_{R}}}\right)^{2}\right]
$$

where now $m_{t}=\left\langle s_{h} c_{h}\right\rangle\left|M_{1}^{t}(0)\right| / \sqrt{16 \Pi_{0}^{t_{L}}(0) \Pi_{0}^{t_{R}}(0)}$ and the correlators are the same as Eq. 24$\rangle$ but

\footnotetext{
${ }^{4}$ As in Ref. [6], we are not considering invariants formed by contracting the spurions with the Levi-Civita tensor (see Appendix B). These invariants can be eliminated by imposing extra parities in the strong sector, along the lines of the models in Ref. [11].
} 
with the replacement $Q_{1} \rightarrow Q_{6}$, since $\mathbf{1 0}=\mathbf{4} \oplus \mathbf{6}$ under $\mathrm{SO}(4)$. For the Higgs mass we obtain Eq. (25) with the replacement $\left(\Delta F^{2}\right)^{2} \rightarrow\left(\Delta F^{2}\right)^{2}+\left|F_{Q_{4}}^{L} F_{Q_{4}}^{R}\right|^{2}$ where now $\Delta F^{2}=\left(3\left|F_{Q_{4}}^{L}\right|^{2}-\left|F_{Q_{4}}^{R}\right|^{2}\right) / 2$. In this model as well, $\left\langle s_{h}\right\rangle \ll 1$ requires $\Delta F^{2}$ small, implying that the Higgs mass in the $\mathrm{MCHM}_{10}$ is always larger than that in the $\mathrm{MCHM}_{5}$. This is shown in Figure 1 .

We then conclude that a light Higgs requires a fermionic resonance mass smaller than the vector one $m_{\rho}$ that, due to constraints from electroweak precision tests [12], must lie above $2-3 \mathrm{TeV} 5$. A possible natural explanation for this mass splitting in the resonance spectrum can be found in holographic models [6]. In these models one finds that in the limit in which $t_{L}$ (or $t_{R}$ ) has large mixings with the composite sector, $\epsilon \sim 1$, extra massive resonances, called custodians, become light and complete with $t_{L}$ (or $t_{R}$ ) a full $\mathrm{SO}(5)$ multiplet. This phenomenon can be understood in the following way. The mixing of the top with the strong-sector resonances depends on the dimension of the chiral fermionic operator to which the top couples to (see Eq. 46) in Appendix A). This mixing becomes more sizable as we decrease the dimension of this operator; when this dimension approaches the lower- bound 3/2, the fermionic operator becomes a free field that decouples from the strong sector and then it mass tends to zero. In the $\mathrm{MCHM}_{5}$ for a $t_{L}$ with sizable mixings $\epsilon$, the light resonances are those states that complete with $t_{L}$ a 5 of $\mathrm{SO}(5)$ [6]: colored states with quantum numbers $\mathbf{1}_{\mathbf{2} / \mathbf{3}}$ and $\mathbf{2}_{\mathbf{7} / \mathbf{6}}$ under the $\mathrm{SM} \mathrm{SU}(2)_{L} \times \mathrm{U}(1)_{Y}$. Similarly, for a $t_{R}$ with large mixing $\epsilon$, these states correspond to two weak-doublets, $\mathbf{2}_{\mathbf{1} / \mathbf{6}}$ and $\mathbf{2}_{\mathbf{7} / \mathbf{6}}$, forming together a $\mathbf{4}$-plet of $\mathrm{SO}(4)$. This connection between large mixings and light resonances has also been studied in simple models of partly composite top [13] or, more recently, via deconstructed versions of the MCHM [7, 8].

\subsection{Extension to other representations}

It is important to analyze whether the fact that a light Higgs implies fermionic resonances below the $\mathrm{TeV}$ goes beyond the minimal realizations $\mathrm{MCHM}_{5,10}$. In this section we will consider the $\mathrm{SO}(5) / \mathrm{SO}(4)$ model with the left-handed and right-handed top quarks embedded respectively in generic representations $\mathbf{r}_{\mathbf{L}}$ and $\mathbf{r}_{\mathbf{R}}$ of $\mathrm{SO}(5)$. The generalization of Eq. (9) can be written in this case as

$$
\begin{aligned}
\mathcal{L}_{\mathrm{eff}} & =\bar{b}_{L} \not p\left(\sum_{i} c_{h}^{i} \Pi_{i}^{b_{L}}(p)\right) b_{L}+\bar{t}_{L} \not p\left(\sum_{i} c_{h}^{i} \Pi_{i}^{t_{L}}(p)\right) t_{L}+\bar{t}_{R} \not p\left(\sum_{i} c_{h}^{i} \Pi_{i}^{t_{R}}(p)\right) t_{R} \\
& +\left(s_{h}^{1+2 m} c_{h}^{n} \bar{t}_{L} M_{1}^{t}(p) t_{R}+\text { h.c. }\right),
\end{aligned}
$$

where $i, m, n$ are positive integers. Some examples are given in Appendix B. In Eq. (27) we have included a contribution from $b_{L}$ that can also be present for some embeddings. In order to guarantee the absence of flavor-violation, we have assumed that only one operator is responsible for the fermion

\footnotetext{
${ }^{5}$ At the one-loop level the fermionic resonances can contribute to electroweak observables. The precise magnitude of these contributions depend on the details of the composite models and will not be discussed here.
} 
masses [11]. In this section we will only consider models with $m=0$ which, as we will show in the next section, can be preferred by the experimental data if a light Higgs with SM-like couplings were confirmed.

One of the most important differences between the $\mathrm{MCHM}_{5}$ and these generic models is that the Higgs mass squared can arise at $O\left(\epsilon^{2}\right)$ (i.e., $m_{h}^{2} \sim \int d^{4} p \Pi_{i>0}$ ), instead of $O\left(\epsilon^{4}\right){ }^{6}$. This can happen when at least two terms with different powers of $c_{h}$ appear in the effective kinetic terms of $t_{L, R}, b_{L}$ (first line of Eq. (27)), generating then a potential $V(h)=\alpha c_{h}^{i}+\beta c_{h}^{j}+\cdots(i \neq j)$ with both $\alpha$ and $\beta$ of $O\left(\epsilon^{2}\right)$. The interesting feature of these scenarios is that a nontrivial minimum with $\left\langle s_{h}\right\rangle \ll 1$ can be accommodated more easily than in the $\mathrm{MCHM}_{5}$ where Eq. (16) requires $\alpha<\beta$ and $O\left(\epsilon^{2}\right)$-terms to be smaller than $O\left(\epsilon^{4}\right)$-terms.

Although this type of models can be thought to be more natural than the $\mathrm{MCHM}_{5}$, they generically predict a heavier Higgs and are therefore disfavored by the present data that hints towards a light Higgs. To quantify this, let us consider the case $\mathbf{r}_{\mathbf{L}}=\mathbf{1 4}$ and $\mathbf{r}_{\mathbf{R}}=\mathbf{1}$. The Higgs potential has the same form as Eq. 15 where now

$$
\beta \simeq 2 N_{c} \int \frac{d^{4} p}{(2 \pi)^{4}} \frac{\Pi_{4}^{t_{L}}}{\Pi_{0}^{t_{L}}}
$$

with $\Pi_{4}^{t_{L}}=5 \Pi_{Q_{1}}^{L} / 4-2 \Pi_{Q_{4}}^{L}+3 \Pi_{Q_{9}}^{L} / 4$ (recall that under $\mathrm{SO}(4), \mathbf{1 4}=\mathbf{9} \oplus \mathbf{4} \oplus \mathbf{1}$ and there are therefore three types of resonances $Q_{1,4,9}^{(n)}$ entering in $\Pi_{4}^{t_{L}}$; see Appendix B). To obtain a finite $\beta$ we need to consider at least 3 resonances, $Q_{1}^{(1)} \equiv Q_{1}, Q_{4}^{(1)} \equiv Q_{4}$ and $Q_{9}^{(1)} \equiv Q_{9}$ that, after imposing the Weinberg sum-rules, gives (for $m_{Q_{4}}^{2} \neq m_{Q_{9}}^{2}$ )

$$
\Pi_{4}^{t_{L}}=\frac{5}{4}\left|F_{Q_{1}}^{L}\right|^{2} \frac{\left(m_{Q_{4}}^{2}-m_{Q_{1}}^{2}\right)\left(m_{Q_{9}}^{2}-m_{Q_{1}}^{2}\right)}{\left(p^{2}+m_{Q_{1}}^{2}\right)\left(p^{2}+m_{Q_{4}}^{2}\right)\left(p^{2}+m_{Q_{9}}^{2}\right)}
$$

and a Higgs mass

$$
m_{h}^{2} \simeq \frac{N_{c}}{\pi^{2}} \frac{5\left|F_{Q_{1}}^{L}\right|^{2}}{4 f^{2}}\left[m_{Q_{1}}^{2} \log \left(\frac{m_{Q_{1}}^{2}}{m_{Q_{9}}^{2}}\right)+\frac{m_{Q_{4}}^{2}\left(m_{Q_{9}}^{2}-m_{Q_{1}}^{2}\right)}{m_{Q_{9}}^{2}-m_{Q_{4}}^{2}} \log \left(\frac{m_{Q_{9}}^{2}}{m_{Q_{4}}^{2}}\right)\right]
$$

The value of $F_{Q_{1}}^{L}$ is related to the top mass:

$$
m_{t}=\frac{\left|F_{Q_{1}}^{L} \tilde{F}_{Q_{1}}^{R *}\right|}{m_{Q_{1}}}\left\langle c_{h} s_{h}\right\rangle
$$

where $\tilde{F}_{Q_{1}}^{R}$ is the mixing of $Q_{1}$ with $t_{R}$. Therefore $F_{Q_{1}}^{L}$ has a lower bound for a given $\tilde{F}_{Q_{1}}^{R}$ and the smallest value of $m_{h}$ is achieved for the largest value of $\tilde{F}_{Q_{1}}^{R}$, or equivalently, when $t_{R}$ is fully composite. In this case it is simpler to start from the beginning assuming that $t_{R}$ appears as a

\footnotetext{
${ }^{6}$ See Ref. [11] where models with this property were first proposed for the $\mathrm{SO}(6) / \mathrm{SO}(4)$ coset.
} 
massless composite state from the strong sector. The calculation for the Higgs mass is then quite easy. The form factor $\Pi_{4}^{t_{L}}$ has to have a massless pole, corresponding to $t_{R}$, with a residue given by $m_{t}^{2} /\left\langle s_{h}^{2} c_{h}^{2}\right\rangle$. The Weinberg sum-rules require in this case only two massive resonances, for instance $Q_{9}$ and $Q_{4}$, leading to

$$
\Pi_{4}^{t_{L}}=\frac{m_{t}^{2}}{\left\langle s_{h}^{2} c_{h}^{2}\right\rangle} \frac{m_{Q_{9}}^{2} m_{Q_{4}}^{2}}{p^{2}\left(p^{2}+m_{Q_{9}}^{2}\right)\left(p^{2}+m_{Q_{4}}^{2}\right)}
$$

and then a Higgs mass

$$
m_{h}^{2} \simeq \frac{N_{c}}{\pi^{2}}\left[\frac{m_{t}^{2}}{f^{2}} \frac{m_{Q_{4}}^{2} m_{Q_{9}}^{2}}{m_{Q_{4}}^{2}-m_{Q_{9}}^{2}} \log \left(\frac{m_{Q_{4}}^{2}}{m_{Q_{9}}^{2}}\right)\right],
$$

which is the same expression as for the $\mathrm{MCHM}_{5}$, Eq. 25), with $\Delta F^{2}=0$ and $Q_{9}$ playing the role of $Q_{1}$. Thus, a Higgs mass of order $125 \mathrm{GeV}$, implies again light resonances. Nevertheless, in this case, we cannot rely on arguments based on holographic models [6, 13] to naturally expect light resonances. As discussed in the previous section, only in the limit of sizable mixing between the top and the strong sector, holographic models predict light resonances. In models with $\mathbf{r}_{\mathbf{R}}=\mathbf{1}$ there are not custodians associated to $t_{R}$. Furthermore, when $t_{R}$ is fully composite, the $t_{L}$ must have a small mixing to the strong sector to predict the right top mass and therefore its custodians are not expected to be light. We could alternatively reduce the Higgs mass to $\sim 125 \mathrm{GeV}$ by tuning, either by demanding a larger value of $f$, or by requiring cancellations in $\Pi_{4}^{t_{L}}$. This latter possibility can be realized, for instance, in the presence of a certain degree of degeneracy between the resonances of different $\mathrm{SO}(4)$-multiplets (for example in the limit $m_{Q_{9}} \rightarrow m_{Q_{1}}$ in Eq. (29)).

To further explore the relation between the Higgs mass and the resonance mass in generic $\mathrm{SO}(5) / \mathrm{SO}(4)$ models, we would like to derive here a generalization of the Higgs mass lower-bound Eq. (22) under certain reasonable assumptions. To do this we use the fact that in any composite Higgs model, the Higgs mass must receive at least the model-independent contribution arising from the top-mass form-factor $M_{1}^{t}(p)$ that is always sizable since $M_{1}^{t}(0) \propto m_{t}$. From the last term in the lagrangian Eq. (27), this contributes to the Higgs potential

$$
\Delta V_{t}(h)=-\beta_{t} s_{h}^{2} c_{h}^{2 n}
$$

where

$$
\beta_{t}=\frac{N_{c}}{8 \pi^{2}} \frac{m_{t}^{2}}{s_{h}^{2} c_{h}^{2 n}} m_{Q}^{2}, \quad m_{Q}^{2} \equiv 2 \int_{0}^{\infty} p d p\left|\frac{M_{1}^{t}(p)}{M_{1}^{t}(0)}\right|^{2} .
$$

Realistic EWSB requires $v / f \ll 1$, a limit that can be achieved in two different ways. The first possibility is to take a large value of $n$. Indeed, from the minimization of $\Delta V_{t}(h)$, we get $\left\langle s_{h}^{2}\right\rangle=$ $1 /(1+n)$ that tends to zero for $n \rightarrow \infty$. In this minimum, we find

$$
m_{h}^{2} \simeq \frac{N_{c}}{2 \pi^{2}} \frac{m_{t}^{2}}{v^{2}} m_{Q}^{2}
$$


valid for any value of $n$. Eq. (36) corresponds to a Higgs mass a factor $\sqrt{2} f / v$ larger than Eq. (22), implying then resonance masses even lighter than in Eq. (23).

The other possibility to have $v / f \ll 1$ is by adjusting parameters in the potential, as it was the case in all models discussed so far. For example, consider adding to $\Delta V_{t}(h)$ the term $\Delta V^{\prime}=\alpha c_{h}^{m^{\prime}}$. A small $v / f$ can now be obtained if $\alpha \simeq-2 \beta_{t} / m^{\prime}$, giving in this limit 7

$$
m_{h}^{2}=\frac{4 \beta_{t} v^{2}}{f^{4}}\left[1-\frac{m^{\prime}}{2}+2 n+\left(1-\frac{m^{\prime}}{2}+n\left(1+m^{\prime}-3 n\right)\right) \frac{v^{2}}{f^{2}}+\cdots\right] .
$$

From Eq. (37) we can write a lower bound valid for any value of $n$ and $m^{\prime}$ such that $m^{\prime} \neq 4 n+2$ :

$$
m_{h}^{2} \gtrsim \frac{N_{c}}{4 \pi^{2}} \frac{m_{t}^{2}}{f^{2}} m_{Q}^{2},
$$

that is a factor 4 smaller than Eq. (22) and, for a given Higgs mass, allows resonance masses $m_{Q}$ a factor 2 larger. For the particular case $m^{\prime}=4 n+2$, on the other hand, the leading term in Eq. (37) vanishes and the Higgs mass squared is then proportional to $v^{4} / f^{4}$. We then have

$$
m_{h}^{2} \simeq n(1+n) \frac{N_{c}}{2 \pi^{2}} \frac{m_{t}^{2}}{f^{2}} \frac{v^{2}}{f^{2}} m_{Q}^{2}
$$

allowing for even larger resonance masses for $n$ small.

We finish this analysis by pointing out a possible caveat in the argument that has led to the Higgs mass lower-bounds discussed above. It could well be that the contribution to the Higgs potential coming from $\beta_{t}$ is cancelled by other terms in the potential in order to obtain realistic EWSB, not giving then any contribution to the Higgs mass. This occurs, for example, in models with the embeddings $\mathbf{r}_{\mathbf{L}}=\mathbf{1 0}, \mathbf{r}_{\mathbf{R}}=\mathbf{5}$ or viceversa 8 , and $\mathbf{r}_{\mathbf{L}}=\mathbf{5}, \mathbf{r}_{\mathbf{R}}=\mathbf{1}$. Let us consider this latter case. The potential, in the $\epsilon^{2}$-expansion, is given by $V(h)=\alpha s_{h}^{2}+\beta s_{h}^{4}+\cdots$ where

$$
\begin{aligned}
\alpha & =-\left.\beta_{t}\right|_{n=0}-2 N_{c} \int \frac{d^{4} p}{(2 \pi)^{4}}\left(\frac{\Pi_{1}^{t_{L}}}{2 \Pi_{0}^{t_{L}}}\right), \\
\beta & =N_{c} \int \frac{d^{4} p}{(2 \pi)^{4}}\left(\frac{\Pi_{1}^{t_{L}}}{2 \Pi_{0}^{t_{L}}}\right)^{2} .
\end{aligned}
$$

Notice that the contribution from the top-mass form-factor, $\beta_{t}$, enters now in $\alpha$ and not in $\beta$. The minimization of the potential gives $\left\langle s_{h}\right\rangle=\sqrt{-\alpha /(2 \beta)}$ and the Higgs mass is given by $m_{h}^{2} \simeq$ $8 \beta\left\langle s_{h}^{2} c_{h}^{2}\right\rangle / f^{2}$, that does not receive any contribution from $\beta_{t}$. The Higgs mass can then be much smaller than in the models discussed above. For two resonances, we have

$$
m_{h}^{2} \simeq \frac{N_{c}}{4 \pi^{2}} \frac{\left|F_{Q_{1}}^{L}\right|^{4}\left\langle s_{h}^{2} c_{h}^{2}\right\rangle}{f^{2}}\left(\frac{1}{2} \frac{m_{Q_{4}}^{2}+m_{Q_{1}}^{2}}{m_{Q_{4}}^{2}-m_{Q_{1}}^{2}} \log \left(\frac{m_{Q_{4}}^{2}}{m_{Q_{1}}^{2}}\right)-1\right)
$$

7 The other interesting possibility is to add $\Delta V^{\prime}=\alpha s_{h}^{2} c_{h}^{m^{\prime}}$ that, instead of predicting Eq. (37), gives $m_{h}^{2} \simeq$ $\frac{4 \beta_{t} v^{2}}{f^{4}}\left(2 n-m^{\prime}\right)$, leading to a Higgs mass even larger than the lower-bound Eq. 38.

${ }^{8}$ This type of models were first considered in Ref. [14] for the $\mathrm{SO}(6) / \mathrm{SO}(5)$ coset. 


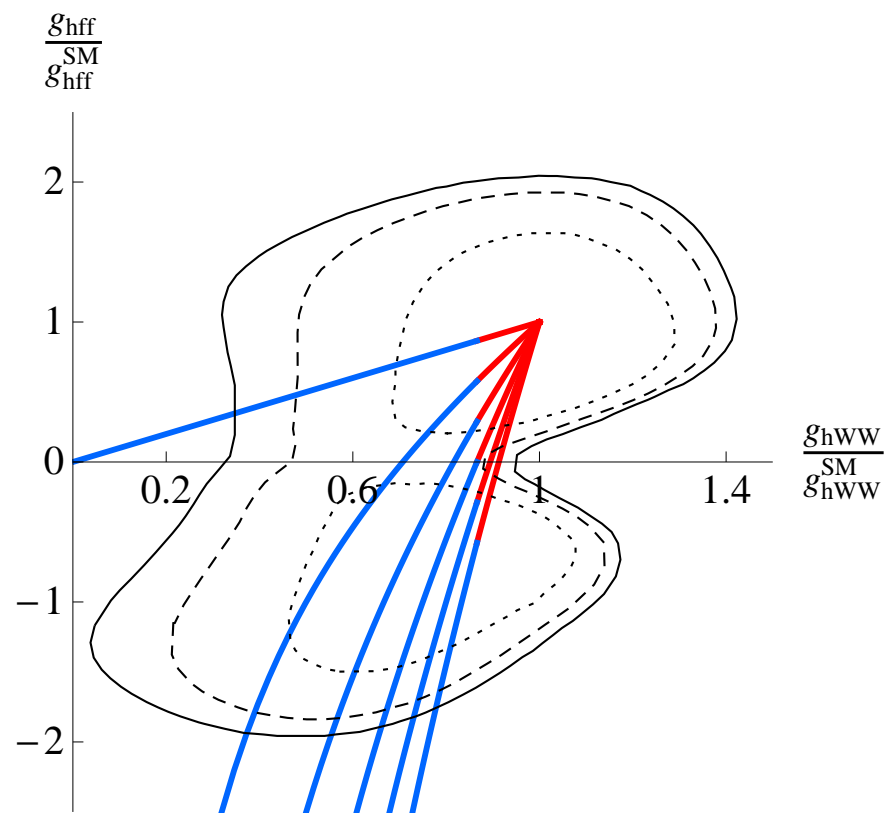

Figure 2: Predictions of a generic MCHM in the $\left(g_{h f f} / g_{h f f}^{\mathrm{SM}}, g_{h W W} / g_{h W W}^{\mathrm{SM}}\right)$-plane. The different curves corresponds to different values of $n$, going downwards from $n=0$ to $n=5$. The red part of the curves is for $0<\xi<0.25$ and the blue one for $0.25<\xi<1$. The contours are the $68 \%, 95 \%$ and $99 \%$ CL for a 125 GeV Higgs as obtained in Ref. [15] from the CMS data.

that is of $O\left(\epsilon^{4}\right)$ and can thus be quite small if $F_{Q_{1}}^{L}$ is small. As in the $\mathbf{r}_{\mathbf{L}}=\mathbf{1 4}, \mathbf{r}_{\mathbf{R}}=\mathbf{1}$ case, the smallest value of $m_{h}$ is found for maximal mixing $\tilde{F}_{Q_{1}}^{R}$ of $t_{R}$ with the composite resonances, i.e. a $t_{R}$ fully composite 9 . Again, it is simpler to start with $t_{R}$ as a massless resonance arising from the strong sector. We then find that one extra massive resonance is enough to satisfy the convergence of the integral in Eq. 41). We have $\Pi_{1}^{t_{L}}(p)=\left(m_{t}^{2} /\left\langle s_{h}^{2}\right\rangle\right) m_{Q_{4}}^{2} /\left(p^{2}\left(p^{2}+m_{Q_{4}}^{2}\right)\right)$ leading to a Higgs mass

$$
m_{h}^{2} \simeq \frac{N_{c}}{8 \pi^{2}} \frac{m_{t}^{4}\left\langle c_{h}^{2}\right\rangle}{v^{2}}\left[\log \left(\frac{m_{Q_{4}}^{2}+m_{t}^{2}}{m_{t}^{2}}\right)-\frac{m_{Q_{4}}^{2}}{m_{Q_{4}}^{2}+m_{t}^{2}}\right] .
$$

For $m_{Q_{4}} \simeq 3 \mathrm{TeV}$, the Higgs mass Eq. 43) can be as small as $40 \mathrm{GeV}$. Larger values of $m_{h}$ imply larger values of $F_{Q_{1}}^{L}$, meaning that $m_{h} \sim 125 \mathrm{GeV}$ can be obtained without light fermionic resonances as we show in Figure 1. In this case, however, it is important to notice that extra contributions are needed to reduce $\alpha$ in order to have $\left\langle s_{h}\right\rangle \ll 1$.

\section{Higgs couplings to SM fermions}

In composite Higgs models the Higgs couplings to fermions generically deviate from their SM values [12]. For the $\mathrm{SO}(5) / \mathrm{SO}(4)$ model, the Higgs couplings to the SM fermions can be parametrized by

${ }^{9}$ The limit of a fully composite $t_{R}$ is also necessary if $\alpha$ of Eq. 40 has to be tuned with the gauge contribution Eq. (7) to guarantee $\left\langle s_{h}\right\rangle \ll 1$. 
Eq. (27). At low-energies $p \ll m_{Q_{i}}$ and in the limit $\epsilon \ll 1$, the Higgs couplings reduce, for the case of a generic SM fermion $f_{L, R}$, to

$$
\mathcal{L}_{\text {eff }} \simeq \bar{f}_{L} M_{1}^{f}(0) f_{R} s_{h}^{1+2 m} c_{h}^{n}+\text { h.c. } \equiv \bar{f}_{L} f_{R} m_{f}(h)+h . c .
$$

From this we can obtain the $h f f$ coupling [12]:

$$
\frac{g_{h f f}}{g_{h f f}^{\mathrm{SM}}}=\frac{2 m_{W}(h)}{g m_{f}(h)} \frac{\partial m_{f}(h)}{\partial h}=\frac{1+2 m-(1+2 m+n) \xi}{\sqrt{1-\xi}},
$$

where we have used that $m_{W}(h)=g s_{h} / 2$ [5] and written the SM $h f f$ coupling as a function of the

physical $W$ and fermion mass, $g_{h f f}^{\mathrm{SM}}=g m_{f} /\left(2 m_{W}\right)$. For $m \neq 0$, Eq. 45 gives deviations of order one from the SM expectations, even in the limit $\xi \rightarrow 1$. For this reason, we will concentrate on the $m=0$ case. In Figure 2 we show, for $m_{h} \simeq 125 \mathrm{GeV}$ and assuming that all fermions couple in the same way, the $68 \%, 95 \%$ and $99 \%$ CL contours for $g_{h f f}$ and $g_{h W W}$ extracted from the most recent CMS data [15] (see also [16] for similar analyses). We have used that the $h W W$ coupling in the $\mathrm{SO}(5) / \mathrm{SO}(4)$ model is given by $g_{h W W}=\sqrt{1-\xi} g_{h W W}^{\mathrm{SM}}$ [12]. Notice that models with large $n$ predict negative values of $g_{h f f}$, and then lie in the lower half-plane of Figure 2, which is also experimentally favored.

An interesting prediction for models where the Higgs potential is dominated by the top-mass term $\Delta V_{t}$, Eq. (34), is that the Higgs is necessarily fermiophobic, corresponding to the horizontal line $g_{h f f}=0$ in Figure 2. This is due to the fact that the minimization condition of the potential implies $\partial m_{t}(h) / \partial h=0$ and then, from Eq. (45), $g_{h f f}=0$.

\section{Discussion and Conclusions}

Using the Weinberg sum-rules in the large- $N$ limit, that require the proper convergence of correlators at large momentum, and using the assumption that these correlators are dominated by the lowestmass resonances, we have computed in the MCHM the mass of the Higgs as a function of the fermionic resonance masses. This has allowed us to show that a light Higgs implies generically light fermionic resonances.

In Figure 3 we give a brief summary of the upper bounds on $m_{Q}$, the lightest fermionic resonance mass, obtained for different classes of MCHM. With an orange line we show the upper bound derived for the $\mathrm{MCHM}_{5}$ [5] and $\mathrm{MCHM}_{10}$ [6] where $m_{h}^{2}$ is of $O\left(\epsilon^{4}\right)$. We can see that a light Higgs, $m_{h} \sim 125$ $\mathrm{GeV}$, implies in this type of models resonances below the TeV. This upper bound on $m_{Q}$ also applies for certain models where $m_{h}^{2}=O\left(\epsilon^{2}\right)$, as for example those with $\mathbf{r}_{\mathbf{L}}=\mathbf{1 4}$ and $\mathbf{r}_{\mathbf{R}}=\mathbf{1}\left(\mathrm{MCHM}_{14}\right)$. The smallest Higgs mass is obtained in this case for a fully composite $t_{R}$, giving the same upper bound on $m_{Q}$ as in the $\mathrm{MCHM}_{5}$. 


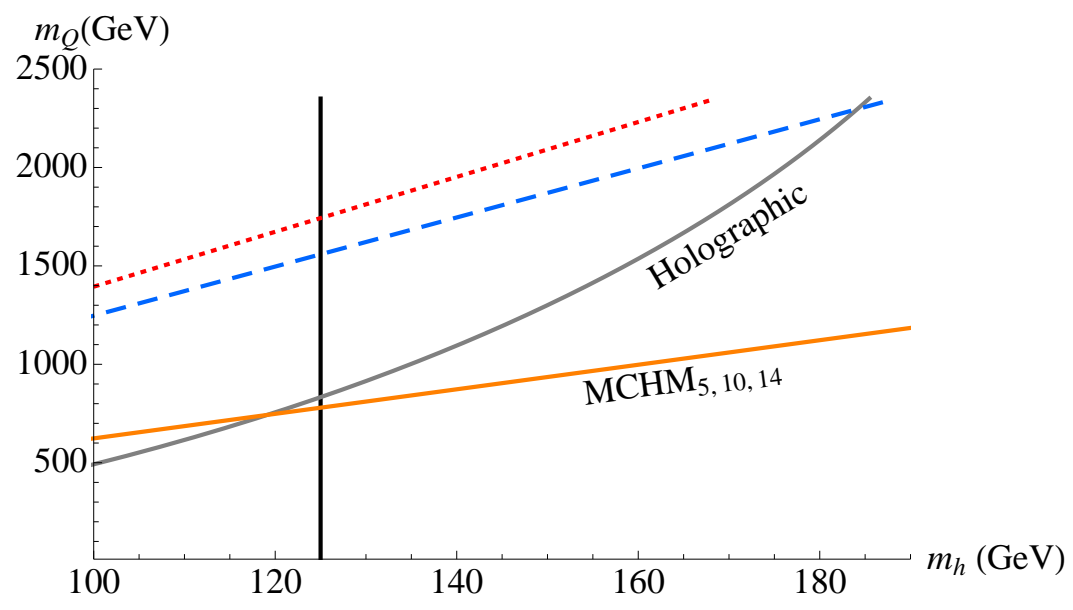

Figure 3: Upper bound for the mass of the lightest fermionic resonance in various composite Higgs models, for $\xi=0.2$ and $m_{t}=160 \mathrm{GeV}$ (the running top mass at $\sim \mathrm{TeV}$ ). The orange line corresponds to Eq. 220) that is the upper bound for the $M C H M_{5,10,14}$. The bound Eq. (38) is shown in dashed blue, while Eq. (39) is shown in dotted red for $n=1$ and $m^{\prime}=6$. A comparison with the Holographic MCHM model of Ref. [6] is shown in grey. The vertical line is for $m_{h}=125 \mathrm{GeV}$.

In Figure 3 we also show in dashed blue the conservative upper-bound Eq. (38), derived under the assumption that the Higgs mass receives contributions from the top form-factor Eq. (34). As we have shown, however, this bound can be evaded in very particular cases; for example, whenever the cancellation needed to reduce the quadratic term in the Higgs potential in order to achieve realistic EWSB, also implies, by accident, a cancellation of the quartic. This can occur at the leading order in $v^{2} / f^{2}$, relaxing the bound on $m_{Q}$ to Eq. (39) (dotted red line in Figure 3 for $n=1$ ), or more drastically to all orders in $v^{2} / f^{2}$ as in models with $\mathbf{r}_{\mathbf{L}}=\mathbf{5}$ and $\mathbf{r}_{\mathbf{R}}=\mathbf{1}$. In this latter case $m_{h}=125 \mathrm{GeV}$ can be obtained even for resonance masses well above $1 \mathrm{TeV}$, as shown by the black line in Figure 1 .

We have studied models based on the minimal coset $\mathrm{SO}(5) / \mathrm{SO}(4)$, but this method can be easily extended to larger cosets [11, 17].

We can then conclude that, with the exception of some particular models, a light Higgs $\sim 125$ $\mathrm{GeV}$ requires generically the presence of fermionic resonances below $m_{\rho} \sim 2-3 \mathrm{TeV}$ that should be possible to discover at the LHC [18]. This also requires that the top should have a sizable degree of compositeness with important collider implications [13, 19].

Note added: The 4th of July 2012, LHC searches [21] have confirmed the presence of a Higgslike state with a mass around $125 \mathrm{GeV}$. An update of Fig. 2 including the new experimental data can be found in Ref. [22]. 


\section{Acknowledgments}

We thank Roberto Contino for providing us the fits of Figure 2. The work of AP was partly supported by the projects FPA2011-25948, 2009SGR894 and ICREA Academia Program.

\section{Appendix A}

In this Appendix we derive the relation between the top-quark form factors $\Pi_{0,1}^{t_{L, R}}, M_{1}^{t}$ and the correlators of the strong sector $\Pi_{Q_{i}}^{L, R}, M_{Q_{i}}$, following Refs. [5, 6]. We do this for the case $\mathbf{r}_{\mathbf{L}, \mathbf{R}}=\mathbf{5}$, and summarize the results for other interesting representations in Appendix B; generalization to other cases is straightforward.

Let us start defining the external sources $\Psi_{L, R}$ that couple to the operators $\mathcal{O}_{L, R}$ of the new strong sector:

$$
\mathcal{L}_{\text {strong }}+\bar{\Psi}_{L} \mathcal{O}_{R}+\bar{\Psi}_{R} \mathcal{O}_{L}
$$

The sources $\Psi_{L, R}$ are in complete $\mathrm{SO}(5)$ multiplets since the lagrangian of the strong sector $\mathcal{L}_{\text {strong }}$, defined in the ultra-violet, is assumed to be $\mathrm{SO}(5)$ invariant. In our particular example $\Psi_{L, R} \in \mathbf{5}$. At low-energies, however, the $\mathrm{SO}(5)$ symmetry is assumed to be spontaneously broken down to $\mathrm{SO}(4)$, and therefore the partition function $\mathcal{Z}$ must be written as a functional of the external sources decomposed, now, into $\mathrm{SO}(4)$ multiplets $Q_{L, R}^{(r)}$, where $r$ labels the $\mathrm{SO}(4)$ representation. In our case, since $\mathbf{5}=\mathbf{4} \oplus \mathbf{1}$ under $\mathrm{SO}(4)$, we write $\Psi_{L, R}=\left(Q_{L, R}^{(4)}, Q_{L, R}^{(1)}\right)$. Integrating over the strong sector, we find the partition function $\mathcal{Z}\left[Q_{L, R}^{(r)}\right]=e^{-\int \mathcal{L}_{\text {eff }}}$ where, at the quadratic order, we have $\mathcal{L}_{\text {eff }}=\bar{Q}_{L}^{(4)} \not p \Pi_{Q_{4}}^{L}(p) Q_{L}^{(4)}+\bar{Q}_{L}^{(1)} \not p \Pi_{Q_{1}}^{L}(p) Q_{L}^{(1)}+(L \rightarrow R)+\bar{Q}_{L}^{(4)} M_{Q_{4}}(p) Q_{R}^{(4)}+\bar{Q}_{L}^{(1)} M_{Q_{1}}(p) Q_{R}^{(1)}+$ h.c. .

The $\Pi_{Q_{r}}^{L}$ are related to the two-point functions of operators of the strong sector according to

$$
\not p \Pi_{Q_{r}}^{L}(p)=\frac{\delta^{2} \mathcal{L}_{\text {eff }}}{\delta \bar{Q}_{L}^{(r)} \delta Q_{L}^{(r)}}=\left\langle\mathcal{O}_{R}^{(r)}(p) \overline{\mathcal{O}}_{R}^{(r)}(-p)\right\rangle .
$$

With the use of the $\mathrm{SO}(5) / \mathrm{SO}(4)$ Goldstones [5]

$$
\Sigma=\frac{s_{h}}{h}\left(h^{(1)}, h^{(2)}, h^{(3)}, h^{(4)}, h \frac{c_{h}}{s_{h}}\right), \quad s_{h} \equiv \sin h / f, \quad h^{2} \equiv \sum_{a=1}^{4}\left(h^{(a)}\right)^{2},
$$

the effective lagrangian Eq. (47) can be written in a $\mathrm{SO}(5)$ invariant way:

$$
\mathcal{L}_{\text {eff }}=\bar{\Psi}_{L}^{i} \not p\left[\delta^{i j} \Pi_{0}^{L}(p)+\Sigma^{i} \Sigma^{j} \Pi_{1}^{L}(p)\right] \Psi_{L}^{j}+(L \rightarrow R)+\bar{\Psi}_{L}^{i}\left[\delta^{i j} M_{0}(p)+M_{1}(p) \Sigma^{i} \Sigma^{j}\right] \Psi_{R}^{j}+h . c .,
$$

where here $i, j=1, \ldots, 5$ label $\mathrm{SO}(5)$ indices. By projecting into the $\mathrm{SO}(4)$-preserving vacuum, $\langle\Sigma\rangle=(0,0,0,0,1)$, we can find the relations between the correlators of Eq. (47) and Eq. (50):

$$
\Pi_{0}^{L, R}=\Pi_{Q_{4}}^{L, R}, \quad \Pi_{1}^{L, R}=\Pi_{Q_{1}}^{L, R}-\Pi_{Q_{4}}^{L, R}, \quad M_{0}=M_{Q_{4}}, \quad M_{1}=M_{Q_{1}}-M_{Q_{4}} .
$$


The couplings of the SM top-quark, $q_{L}=\left(t_{L}, b_{L}\right)$ and $t_{R}$, to the strong sector are defined by their embedding into the external $\mathrm{SO}(5)$-multiplets $\Psi_{L, R}$ since, according to Eq. (46), this tells us to which operators they couple to. For the case $\Psi_{L, R} \in \mathbf{5}$, this is uniquely given by

$$
\Psi_{L}=\frac{1}{\sqrt{2}}\left(b_{L},-i b_{L}, t_{L}, i t_{L}, 0\right), \quad \Psi_{R}=\left(0,0,0,0, t_{R}\right)
$$

Plugging Eq. (52) into Eq. (50), expanding around the vacuum $\Sigma=\left(0,0,0, s_{h}, c_{h}\right)$ (that can be achieved from Eq. (49) after a proper SU(2) rotation) and using Eq. (51), one obtains Eq. (9) and Eq. (11).

\section{Appendix B}

\begin{tabular}{|c||c|c|c|c|}
\hline $\mathbf{r}_{\mathbf{L}} \backslash \mathbf{r}_{\mathbf{R}}$ & $\mathbf{1}$ & $\mathbf{5}$ & $\mathbf{1 0}$ & $\mathbf{1 4}$ \\
\hline \hline $\mathbf{5}$ & $m=n=0$ & $m=0, n=1$ & $m=n=0$ & $m=n=0$ \\
\hline 10 & - & $m=n=0$ & $\begin{array}{c}(i) m=0, n=1 \\
(i i) m=n=0\end{array}$ & $m=0, n=1$ \\
\hline 14 & $m=0, n=1$ & $\begin{array}{c}(i) m=n=0 \\
(i i) m=0, n=2\end{array}$ & $m=0, n=1$ & $\begin{array}{c}(i) m=0, n=1 \\
(i i) m=1, n=1\end{array}$ \\
\hline
\end{tabular}

Table 1: Values of $m, n$ in Eq. (57) for different embeddings.

In this appendix, we list the analog of the effective lagrangian Eq. (9) for different embeddings of $t_{L}$ and $t_{R}$ in $\mathrm{SO}(5)$ representations, $\mathbf{r}_{\mathbf{L}}$ and $\mathbf{r}_{\mathbf{R}}$ respectively (Eq. (9) corresponds to $\mathbf{r}_{\mathbf{L}, \mathbf{R}}=\mathbf{5}$ ).

We split the lagrangian in three parts, $\mathcal{L}_{\text {eff }}=\mathcal{L}_{\text {eff }}^{\mathrm{LL}}+\mathcal{L}_{\text {eff }}^{\mathrm{RR}}+\mathcal{L}_{\text {eff }}^{\mathrm{LR}}$. For the $L L$ and $R R$ part, we have for the $\mathbf{1 0}=\mathbf{6} \oplus \mathbf{4}$ (under $\mathrm{SO}(4)$ ):

$$
\begin{array}{ll}
\mathbf{r}_{\mathbf{L}}=10: & \mathcal{L}_{\mathrm{eff}}^{\mathrm{LL}}=\bar{b}_{L} \not p\left(\Pi_{0}^{b_{L}}+\frac{1}{2} c_{h}^{2} \Pi_{2}^{b_{L}}(p)\right) b_{L}+\bar{t}_{L} \not p\left(\Pi_{0}^{t_{L}}+\left(\frac{1}{2} c_{h}^{2}-\frac{1}{4} s_{h}^{2}\right) \Pi_{2}^{t_{L}}(p)\right) t_{L}, \\
\mathbf{r}_{\mathbf{R}}=\mathbf{1 0}: & \mathcal{L}_{\mathrm{eff}}^{\mathrm{RR}}=\bar{t}_{R} \not p\left(\Pi_{0}^{t_{R}}-2 c_{h} \Pi_{1}^{t_{R}}(p)+\frac{1}{4} s_{h}^{2} \Pi_{2}^{t_{R}}(p)\right) t_{R},
\end{array}
$$

where

$$
\Pi_{0}^{t_{L}, t_{R}}=1+\Pi_{Q_{4}}^{L, R}, \quad \Pi_{1}^{t_{R}}=-\frac{1}{2} \tilde{\Pi}_{Q_{6}}^{R}, \quad \Pi_{2}^{t_{L}, t_{R}}=2\left(\Pi_{Q_{6}}^{L, R}-\Pi_{Q_{4}}^{L, R}\right),
$$

and $\Pi_{0,2}^{b_{L}}=\Pi_{0,2}^{t_{L}}$. We have included a term contracted with the Levi-Civita tensor that in the corresponding of Eq. 47) reads, $\bar{Q}_{R i j}^{(6)} \not p \tilde{\Pi}_{Q_{6}}^{R}(p) Q_{R k l}^{(6)} \epsilon^{i j k l}$. For the $\mathbf{1 4}=\mathbf{9} \oplus \mathbf{4} \oplus \mathbf{1}$, we have

$$
\begin{aligned}
& \mathbf{r}_{\mathbf{L}}=14: \mathcal{L}_{\mathrm{eff}}^{\mathrm{LL}}=\bar{b}_{L} \not p\left(\Pi_{0}^{b_{L}}+\frac{1}{2} c_{h}^{2} \Pi_{2}^{b_{L}}(p)\right) b_{L}+\bar{t}_{L} \not p\left(\Pi_{0}^{t_{L}}+\left(\frac{1}{2} c_{h}^{2}-\frac{1}{4} s_{h}^{2}\right) \Pi_{2}^{t_{L}}(p)+s_{h}^{2} c_{h}^{2} \Pi_{4}^{t_{L}}(p)\right) t_{L}, \\
& \mathbf{r}_{\mathbf{R}}=\mathbf{1 4}: \mathcal{L}_{\mathrm{eff}}^{\mathrm{RR}}=\bar{t}_{R} \not p\left(\Pi_{0}^{t_{R}}+\left(\frac{4}{5} c_{h}^{2}+\frac{1}{20} s_{h}^{2}\right) \Pi_{2}^{t_{R}}(p)+\frac{1}{20}\left(4 c_{h}^{2}-s_{h}^{2}\right)^{2} \Pi_{4}^{t_{R}}(p)\right) t_{R},
\end{aligned}
$$


where now

$$
\Pi_{0}^{t_{L}, t_{R}}=1+\Pi_{Q_{9}}^{L, R}, \quad \Pi_{2}^{t_{L}, t_{R}}=2\left(\Pi_{Q_{4}}^{L, R}-\Pi_{Q_{9}}^{L, R}\right), \quad \Pi_{4}^{t_{L}, t_{R}}=\frac{5}{4} \Pi_{Q_{1}}^{L, R}-2 \Pi_{Q_{4}}^{L, R}+\frac{3}{4} \Pi_{Q_{9}}^{L, R},
$$

and, as before, $\Pi_{0,2}^{b_{L}}=\Pi_{0,2}^{t_{L}}$. For the $L R$ terms we have

$$
\mathcal{L}_{\mathrm{eff}}^{\mathrm{LR}}=s_{h}^{1+2 m} c_{h}^{n} \bar{t}_{L} M_{1}^{t}(p) t_{R}+\text { h.c. },
$$

where the values of $m$ and $n$ are given in Table 1 . In cases where two terms with different values of $m, n$ are possible, one of the two must be suppressed to avoid large flavor-violations; this can be achieved by imposing extra parities to the strong sector, as advocated in Ref. [1].

\section{References}

[1] G. Aad et al. [ATLAS Collaboration], Phys. Lett. B 710 (2012) 49 [arXiv:1202.1408 [hep-ex]].

[2] S. Chatrchyan et al. [CMS Collaboration], Phys. Lett. B 710 (2012) 26 arXiv:1202.1488 [hep$\mathrm{ex}]]$.

[3] R. Barate et al. [LEP Working Group for Higgs boson searches and ALEPH and DELPHI and L3 and OPAL Collaborations], Phys. Lett. B 565 (2003) 61 hep-ex/0306033.

[4] D. B. Kaplan and H. Georgi, Phys. Lett. B 136 (1984) 183; H. Georgi and D. B. Kaplan, Phys. Lett. B 145 (1984) 216.

[5] K. Agashe, R. Contino and A. Pomarol, Nucl. Phys. B 719 (2005) 165 hep-ph/0412089].

[6] R. Contino, L. Da Rold and A. Pomarol, Phys. Rev. D 75 (2007) 055014 hep-ph/0612048.

[7] G. Panico and A. Wulzer, JHEP 1109 (2011) 135 arXiv:1106.2719 [hep-ph]]; O. Matsedonskyi, G. Panico and A. Wulzer, arXiv:1204.6333 [hep-ph].

[8] S. De Curtis, M. Redi and A. Tesi, JHEP 1204 (2012) 042 [arXiv:1110.1613 [hep-ph]]; M. Redi and A. Tesi, arXiv:1205.0232 [hep-ph].

[9] S. Weinberg, Phys. Rev. Lett. 18 (1967) 507.

[10] T. Das, G. S. Guralnik, V. S. Mathur, F. E. Low and J. E. Young, Phys. Rev. Lett. 18 (1967) 759.

[11] J. Mrazek, A. Pomarol, R. Rattazzi, M. Redi, J. Serra and A. Wulzer, Nucl. Phys. B 853 (2011) 1 [arXiv:1105.5403 [hep-ph]]. 
[12] G. F. Giudice, C. Grojean, A. Pomarol and R. Rattazzi, JHEP 0706 (2007) 045 hepph/0703164.

[13] A. Pomarol and J. Serra, Phys. Rev. D 78 (2008) 074026 [arXiv:0806.3247 [hep-ph]].

[14] M. Frigerio, A. Pomarol, F. Riva and A. Urbano, arXiv:1204.2808 [hep-ph].

[15] A. Azatov, R. Contino and J. Galloway, JHEP 1204 (2012) 127 [arXiv:1202.3415 [hep-ph]].

[16] D. Carmi, A. Falkowski, E. Kuflik and T. Volansky, arXiv:1202.3144 [hep-ph]; J. R. Espinosa, C. Grojean, M. Muhlleitner and M. Trott, arXiv:1202.3697 [hep-ph].

[17] B. Gripaios, A. Pomarol, F. Riva and J. Serra, JHEP 0904 (2009) 070 arXiv:0902.1483 [hepph]].

[18] See for example, R. Contino and G. Servant, JHEP 0806 (2008) 026 arXiv:0801.1679 [hepph]]; J. A. Aguilar-Saavedra, JHEP 0911 (2009) 030 [arXiv:0907.3155 [hep-ph]]; J. Mrazek and A. Wulzer, Phys. Rev. D 81 (2010) 075006 [arXiv:0909.3977 [hep-ph]]; G. Dissertori, E. Furlan, F. Moortgat and P. Nef, JHEP 1009 (2010) 019 [arXiv:1005.4414 [hep-ph]].

[19] B. Lillie, J. Shu and T. M. P. Tait, JHEP 0804 (2008) 087 [arXiv:0712.3057 [hep-ph]].

[20] D. Marzocca, M. Serone and J. Shu, arXiv:1205.0770 [hep-ph].

[21] J. Incandela, CMS talk at Latest update in the search for the Higgs boson at CERN, July 4, 2012; F. Gianotti, ATLAS talk at Latest update in the search for the Higgs boson at CERN, July 4, 2012.

[22] M. Montull and F. Riva, arXiv:1207.1716 [hep-ph]. 